
$\left(\mathrm{XV}^{\mathrm{e}}-\mathrm{XIX}{ }^{\mathrm{e}}\right.$ siècle)

\title{
Se substituer à Ptolémée ? Modèles, sources et mise en forme de la géographie dans les Navigationi et viaggi de Giovanni Battista Ramusio
}

Fiona Lejosne

\section{OpenEdition}

\section{Journals}

Édition électronique

URL : https://journals.openedition.org/geohist/314

DOI : 10.4000/geohist.314

ISSN : 2264-2617

Éditeur

Association française de la Revue de géographie historique

Référence électronique

Fiona Lejosne, «Se substituer à Ptolémée? Modèles, sources et mise en forme de la géographie dans les Navigationi et viaggi de Giovanni Battista Ramusio », Revue de géographie historique [En ligne],

17-18 | 2020, mis en ligne le 03 novembre 2020, consulté le 12 juin 2021. URL : http:// journals.openedition.org/geohist/314; DOI : https://doi.org/10.4000/geohist.314

Ce document a été généré automatiquement le 12 juin 2021.

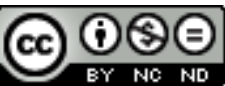

Ce(tte) œuvre est mise à disposition selon les termes de la Licence Creative Commons Attribution -

Pas d'Utilisation Commerciale - Pas de Modification 4.0 International. 


\title{
Se substituer à Ptolémée ? Modèles, sources et mise en forme de la géographie dans les Navigationi et viaggi de Giovanni Battista Ramusio
}

\author{
Fiona Lejosne
}

\section{Introduction}

1 Les discours de définition et de légitimation de la géographie dans les Navigationi et viaggi, compilation géographique publiée au milieu du XVI siècle, constituent l'objet de notre étude. Il s'agit de l'œuvre d'un géographe vénitien, Giovanni Battista Ramusio, qui rassemble en trois volumes près de soixante textes d'écrivains anciens comme modernes. Ces textes sont assortis d'introductions et de commentaires, les discorsi, dans lesquels Ramusio expose les motifs des choix de ces textes et apporte des informations concernant leur auteur ou le contexte du voyage qui y est raconté. Ces discorsi confèrent à la compilation sa dimension organique puisque, d'une part, ils créent une structure dans laquelle est contenu son matériau disparate, et que, d'autre part, ils sont l'occasion pour Ramusio de mettre en évidence les liens entre les informations rapportées par chacun des textes recueillis. L'analyse du discours sur le savoir géographique de ces discorsi fait donc partie intégrante d'une étude des Navigationi et viaggi : à la fois parce que Ramusio propose une forme originale d'organisation du savoir géographique et parce que sa propre contribution à la compilation consiste justement en la rédaction de ces discorsi introductifs, des para-textes propices à l'explicitation de sa conception du savoir géographique.

Dans la dédicace du premier volume, le constat du caractère obsolète des cartes de Ptolémée est désigné comme l'élément déclencheur du projet. Ainsi Ramusio affirme-til que « la raison pour laquelle [il] besogn[a] volontiers pour cette œuvre » s'explique par le fait qu'il « v[it] et considér[a] que les tables de la Géographie de Ptolémée où sont 
décrites l'Afrique et l'Inde étaient très imparfaites ». Ce constat d'une désuétude des cartes de Ptolémée est rendu évident par la comparaison entre leur contenu et «la grande connaissance que l'on a aujourd'hui de ces régions» (Ramusio, 1554 n.p.). Ramusio identifie plus loin dans le premier volume la seconde cause de l'obsolescence de Ptolémée, dans le discorso introductif aux récits des navigations portugaises vers les Indes orientales qui commence par ces mots : « une des choses les plus grandes et les plus admirables qu'ait vue notre époque est la découverte de tant de pays différents et variés, que jamais auparavant nos anciens n'avaient connus » (Ramusio, 1554 fol. 129v.). Pour un homme comme Ramusio, lecteur attentif des auteurs classiques de langues grecque et latine, et géographe curieux et soucieux de s'informer des dernières découvertes de son temps, la comparaison entre les deux ne pouvait se faire qu'à la faveur des Modernes : non seulement ceux-ci connaissent des parties du monde que les Anciens ne connaissaient pas, mais, en plus, ils ont conduit des explorations qui leur ont permis de contredire certaines représentations ou hypothèses élaborées par leurs lointains prédécesseurs.

Dès lors, l'ambition d'une compilation comme celle de Ramusio ne peut être que de se substituer à Ptolémée: si lui-même ne s'en targue pas de manière ostentatoire, il affirme toutefois que les quelques cartes ajoutées en tête du premier volume dans la version de 1554 devraient suffire à ce qu'«il ne devienne que peu nécessaire de besogner dur sur les tables de Ptolémée » (Ramusio, 1554 n.p., après l'index). Tommaso Giunti, l'éditeur de la compilation, se charge de formuler explicitement cette ambition de suppléance des Anciens. Dans une adresse aux lecteurs également insérée dans la deuxième édition du premier volume, il souligne que, si le projet des Navigationi et viaggi était mené à terme, «il ne serait plus nécessaire de lire ni Ptolémée, ni Strabon, ni Pline, ni aucun autre auteur ancien en matière de Géographie » (Ramusio, 1554 n.p.). Cette remarque identifie ainsi d'autres modèles qui ont pu présider à la constitution du recueil, à côté de celui de l'auteur de la Géographie. Elle ne peut être prise pour argent comptant puisqu'elle apparaît dans le contexte d'une adresse aux lecteurs à valeur promotionnelle; toutefois, elle met le doigt sur une ambition qui transparaît réellement dans nombre des discorsi de Ramusio : celle de se substituer à la fois à une géographie de la quantité, c'est-à-dire faite selon le mathematicorum more (comme Ptolémée), et à une géographie de la qualité, réalisée en fonction de l'historicum more (comme Strabon et Pline).

Remarquons par ailleurs que ce projet de substitution diffère d'entreprises contemporaines des Navigationi et Viaggi, qui consistaient par exemple en une remise à jour de la Géographie par augmentation (pour les terres nouvellement découvertes) ou par modification lorsque les informations rassemblées par Ptolémée étaient devenues obsolètes (Milanesi, 1984). Le recueil des Navigationi et viaggi ne reprend pas tel quel le cadre de la Géographie dans la mesure où il s'agit d'une compilation de récits de voyages et de descriptions géographiques, c'est-à-dire de textes rendant compte de l'expérience des hommes dans le monde. Une telle œuvre participe plutôt de la géographie qualitative : en racontant les observations effectuées au cours d'un voyage, les auteurs disent le monde en le décrivant. De plus, un récit de voyage concerne nécessairement des aires géographiques limitées puisqu'il ne rapporte que les constats liés au déplacement d'un seul homme. Il semblerait ainsi que les textes recueillis dans les Navigationi et Viaggi appartiennent à la chorographie plutôt qu'à la géographie. En effet, selon les catégories énoncées ou précisées au XVI e siècle (Milanesi, 1994 et Tessicini, 2011), la première étudie son objet à grande échelle, et l'aborde par la description, se 
distinguant ainsi de la seconde qui, toujours par la description, s'intéresse au monde à petite échelle. De plus, si l'on reprend la distinction établie par exemple par Pierre Apian, la cosmographie partage avec la géographie l'échelle prise en compte, mais s'en distingue au sens où elle ne vise pas à décrire le monde mais à en rendre compte par des mesures (Hallyn, 2008). Dès lors, il convient de se demander comment le projet de se substituer aux géographes anciens est mis en œuvre par Ramusio: par quelles sources, avec quelle mise en forme et selon quels modèles?

\section{L'expérience comme source du savoir}

5 Si l'emploi de récits de voyage comme source de la géographie n'est pas une nouveauté en soi (Bouloux, 2002), il faut toutefois noter le choix radical opéré par Ramusio: le recueil Navigationi et viaggi est constitué pour ses neuf dixièmes de récits de voyage. Ce choix n'est jamais explicitement commenté dans les dicorsi, toutefois, deux revendications nous permettent de comprendre les motifs de cette sélection: la conviction d'une source expérimentale du savoir géographique d'une part, et l'attention portée à l'authenticité des textes d'autre part.

6 En ce qui concerne la nécessité pour la géographie de prendre appui sur l'expérience des hommes dans le monde, les affirmations de Ramusio ne peuvent laisser place à l'équivoque. En effet, il souligne à de nombreuses reprises que les témoignages des voyageurs modernes apportent constamment des démentis au savoir géographique préétabli. Que ceux-ci aient été mal informés ou qu'ils aient fait des conjectures sur des zones qui n'avaient pas encore été explorées, les Anciens se sont souvent trompés et les explorations des Modernes permettent de démasquer leurs erreurs. Ainsi, l'expérience moderne du voyage est systématiquement associée à l'idée d'une révélation de la vérité. Dans le discorso sur les crues du Nil, l'opposition est nettement marquée entre «la certitude de l'expérience» et «la probabilité des causes» (Ramusio, 1554 fol. 287v.-288r.). De même, dans le discorso sur la navigation en mer Rouge d'Arrien, l'emploi des termes «vérité " et "vrai » est exclusivement réservé aux commentaires sur les explorations modernes des Portugais : il est ainsi fait référence à « la vérité que l'on connaît maintenant grâce aux navigations portugaises » et à ce que "aujourd'hui l'on sait pour sûr, grâce à la vraie relation de qui s'est rendu en ces lieux » (1554 fol. 312r.). Dans l'introduction au deuxième volume, la notion de révélation de la vérité est d'ailleurs associée à celle de "vrai témoignage ", mettant ainsi en évidence la double sémantique de ce terme: le témoin, celui qui a vécu le voyage et qui a fait l'expérience de ce qui est rapporté par le récit, est également celui qui est cité comme preuve de cette nouvelle vérité (Ramusio, 1559 fol. 17v.). En d'autres termes, c'est parce que le voyageur a voyagé qu'il constitue une source fiable du savoir. Dans ce cadre, il est donc évident que, pour offrir une géographie dont le contenu soit satisfaisant au vue des explorations alors récentes ou en cours, Ramusio se doit de livrer des textes rendant compte directement de ces expériences, c'est-à-dire des récits de voyage. Quel que soit le statut énonciatif de ceux-ci - qu'ils aient été écrits par le voyageur lui-même ou non, qu'ils aient été écrits au cours du voyage ou après celui-ci - ces récits procurent en effet une description du monde qui ne résulte pas de la conjecture mais de l'observation.

7 À cette conviction que le savoir géographique doit se fonder sur l'expérience s'ajoute chez Ramusio une préoccupation typiquement humaniste : le texte doit être livré dans 
sa forme la plus authentique possible. Dans le cas du récit de voyage, le risque d'une distorsion ou d'un appauvrissement du texte d'origine est fort. D'une part, les témoignages ne sont pas toujours considérés à leur juste valeur et peuvent donc être facilement perdus (Ramusio assigne à son recueil un rôle de conservation et de divulgation, par exemple dans le cas du récit de Marco Polo); d'autre part, les récits de voyage encourent des risques de modifications volontaires du texte dus aux enjeux politiques ou commerciaux avec lesquels ils sont susceptibles d'interférer. Ce risque est identifié comme étant particulièrement élevé dans le cas des récits concernant l'aire sous domination portugaise (Lejosne, 2017). Ainsi, Ramusio revendique la nécessité d'avoir recours aux récits d'expérience tout en constatant que ceux-ci peuvent contenir des inexactitudes résultant de la détérioration de leur vecteur de diffusion, le texte. Une partie de son rôle en tant que compilateur consiste en l'édition de ces textes : les discorsi décrivent en effet un minutieux travail de recherche de différentes versions, ainsi que de collation et de correction des textes ainsi obtenus.

8 Ce travail philologique d'empreinte humaniste - c'est-à-dire ce processus de préparation du texte pour le consigner à la postérité dans sa leçon considérée comme la plus correcte possible - suppose donc que l'on attribue au texte lui-même un haut degré de fiabilité qui peut être restitué par l'émendation des copies fautives. Cependant, si le récit de voyage est retenu comme le matériau principal de la géographie, se pose toutefois la question de la fiabilité de celui-ci indépendamment des conditions de sa publication: qu'est-ce qui fait que l'expérience qu'il rapporte est considérée comme porteuse de vérité? Comment s'assurer que cette source est suffisante pour élaborer un savoir géographique satisfaisant? De plus, le problème de la garantie du contenu du récit de voyage est doublé d'une autre limite intrinsèque à cette source : les bornes de sa représentation spatiale. En effet, le récit de voyage, on l'a dit, ne rend compte que de l'expérience d'un espace limité. Dans un cas comme dans l'autre, nous allons voir de quelle manière la résolution de ces difficultés est permise par la mise en forme choisie, la compilation.

\section{La compilation : accumulation d'expériences}

9 Les discorsi introductifs ne taisent pas les limites de chacun des textes recueillis, au contraire, ils les mettent généralement en évidence : il peut s'agir de la couverture spatiale du voyage en question lorsque, à l'instar de Francesco Alvarez et des sources du Nil, le voyageur n'est pas allé aussi loin que ce que les géographes auraient souhaité (Ramusio, 1554 fol. 208r.) ou de la capacité d'observation de l'individu. Pour cette raison, souligne Ramusio, il est nécessaire de lire plusieurs textes traitant d'un même espace. De plus, la contradiction entre deux sources recueillies est une possibilité explicitement prise en compte par Ramusio dans ses commentaires. Cependant, la forme compilatoire est présentée dans les discorsi comme le facteur d'une complémentarité entre ces expériences de voyage: l'accumulation de textes traitant d'un même espace permet d'obtenir un savoir satisfaisant sur cet espace.

Les expériences personnelles de parcours et de connaissance du monde qui sont à l'origine des récits de voyage ont pour base l'observation des voyageurs: or, rappelle Ramusio, les hommes ne sont pas tous attentifs aux mêmes choses et n'utilisent pas tous leurs sens de la même manière. En conséquence, des différences pourront être constatées d'un récit à l'autre. Ainsi, dans le discorso sur la circumnavigation du globe il 
remarque que «les esprits des hommes sont divers, qui note une chose, qui en note une autre, selon celles qui leur semblent les plus dignes » (Ramusio, 1554 fol. 383r.). C'est donc dans ce risque d'une divergence que se trouve tout l'intérêt de la compilation: elle permet d'avoir à disposition, dans le même espace-livre, divers comptes rendus d'expérience et elle donne ainsi aux lecteurs l'occasion de confronter par eux-mêmes ces expériences. Ramusio justifie de la sorte le fait d'avoir choisi deux récits fort similaires dans le cas de la circumnavigation du globe, soit ceux d'Antonio Pigafetta et de Maximilianus Transylvanus. En effet, il remarque que « de nombreuses parties, qui par l'un ont été laissées de côté, peuvent être lues abondamment chez l'autre » (ibid.). Le même argument des variantes individuelles est avancé dans le discorso sur le récit d'Hayton l'Arménien : «si le fil de cette histoire n'est pas aussi continu qu'il devrait l'être, que les lecteurs fassent preuve de clémence, puisque les hommes n'ont pas pour habitude de tous raconter une même chose de la même façon, mais de manière variée selon la diversité de leurs esprits » (1559 fol. 61r.). Ramusio indique parfois les raisons pour lesquelles on peut préférer la lecture d'un texte à un autre, par exemple si l'on se réfère à la quantité de détails fournis : ainsi, dans la description conjointe des lettres de Andrea Corsali et du récit de Francisco Álvares sur l'Ethiopie, le récit du second (bien qu'ayant été obtenu en version incomplète) se distingue pour la précision bien supérieure de son contenu (Ramusio, 1554 fol. 193v.).

11 Les différences entre deux récits portant sur un même espace, voire les éventuelles discordances entre ceux-ci, rendent la compilation nécessaire en vue de l'élaboration d'un savoir géographique fiable. On remarque toutefois que, dans ses para-textes, Ramusio dit ne pas vouloir opérer de sélection lorsqu'il y a divergence entre les textes édités; il les livre tels quels et s'en remet aux lecteurs qui doivent eux-mêmes procéder à ce tri par la lecture comparée des récits recueillis. Au lieu de se soustraire aux contradictions possibles, la compilation les contient et les expose, afin de laisser à d'autres la possibilité de les résoudre. Cette résolution peut d'ailleurs faire appel à des données encore inaccessibles au moment où Ramusio publie ses volumes, ou encore à des vérifications à effectuer in situ. La forme compilatoire se présente donc à la fois comme un chantier en cours, un ouvrage que des explorations prochaines vont doivent - compléter, et comme un travail préalable à l'élaboration d'un savoir construit et organisé. Le compilateur se présente en effet dans les discorsi comme celui qui met à la disposition des autres.

La forme compilatoire comme garantie d'une accumulation d'expériences dont la lecture et la confrontation permettent d'élaborer un savoir fiable n'est que partiellement satisfaisante pour le géographe : elle permet une connaissance des lieux mais elle ne rend pas nécessairement possible leur positionnement. En effet, les sources choisies et la mise en forme adoptée par Ramusio n'offrent qu'une situation des lieux décrits les uns par rapport aux autres, selon les parcours effectués par les voyageurs et reproduits dans leur récit, ainsi que dans la succession des textes recueillis. L'absence de recours à une organisation topique telle que celle de l'emboîtement, où le point de départ du discours géographique est la totalité, et où la localité est secondaire (Bouloux, 2002 et Besse, 2003), met-elle en péril la possibilité d'une représentation globale ? Nous allons analyser, par la manière dont Ramusio envisage à deux reprises la Terre dans son intégralité, sa conception de la géographie de la Terre. 


\section{Verticalité et dispositif cosmographique}

13 Le discorso d'introduction au troisième volume contient à la fois une démonstration de la supériorité de l'expérience sur les conjectures et une illustration de la nécessité d'avoir parfois recours à ces conjectures. Le début de ce discorso est consacré à la question de l'impact des nouveaux espaces explorés par les Espagnols sur la connaissance de l'œkoumène. Le caractère inattendu de la découverte du continent américain est minimisé par Ramusio dans une démonstration qui a à la fois recours à Platon et à l'exemple des voyages récents - mais antérieurs à ceux inaugurés par Christophe Colomb - effectués par les Européens. En effet, Ramusio souligne que la possibilité de l'habitabilité d'autres zones que la zone tempérée où se trouve l'Europe occidentale a été suggérée par Platon dans le Timée, où est exposé le mythe de l'Atlantide, et a été démontrée par le voyage du Vénitien Pietro Querini lors du naufrage qui le porta jusqu'aux îles Lofoten en janvier 1432 (Ramusio, 1559 fol. 144r.-155v. et Querini, Fioravante, de Michiel, 2005). Les barrières nord et sud de la zone tempérée (le Pôle Nord et le Tropique du Cancer), traditionnellement considérées comme infranchissables par l'homme (Headley, 1997), ont donc été doublées avant même que ne soit explorée par les Espagnols « cette nouvelle partie du monde ». Dans la première partie de ce discorso, le même mécanisme rhétorique que celui des discours précédemment cités est mis en œuvre: Ramusio montre qu'une hypothèse formulée par les Anciens a été infirmée par les explorations des Modernes (Ramusio, 1556 fol. 2v.).

14 Cependant, ce discorso suggère également que certaines observations issues de l'expérience de ces voyageurs ne peuvent être expliquées par leurs seuls récits. En effet, les voyageurs se contentent de constater la différence de saisons, la variation dans la durée des journées et plus généralement la diversité de ce qui se trouve à la surface de la Terre; mais, pour expliquer ces variations, on est contraint à avoir recours à la cosmographie. Pour les saisir, Ramusio invite en effet son lecteur à faire usage d'un dispositif cosmographique qui consiste à se représenter le mouvement du Soleil autour de la Terre :

Cette variété, bien qu'elle paraisse incompréhensible à la petitesse de l'esprit humain, toutefois, en passant par une spéculation par l'œil de l'intellect, et en prenant d'abord en compte le mouvement inestimable que fait continuellement le Soleil, on verra qu'elle est vraie eu égard à la diversité des lieux de la Terre qui continuellement sont illuminés. (Ramusio, 1556 fol. 3r.)

La totalité de la Terre est donc envisagée par le biais d'une médiation cosmographique qui a pour objectif d'expliquer la variété constatée à la surface de celle-ci. Ainsi, la seule fois où Ramusio envisage une représentation totale de la Terre (ce globe autour duquel tourne le Soleil selon la conception qu'il rapporte dans ce discorso) celle-ci répond à un besoin d'explication prenant racine dans l'expérience elle-même, mais que l'expérience ne peut suffire à comprendre. On remarque que cette démarche cognitive ne suit pas le processus cosmographique classique - qui consiste en une projection sur la Terre des astres et de leur influence - puisqu'elle part du constat de la variété à la surface de la Terre pour arriver à l'influence du Soleil sur celle-ci. Toutefois, le caractère abstrait et non expérimental de l'explication est mis en évidence par l'expression «œil de l'intellect ». Ainsi, ce discorso renvoie toute prétention de la géographie à livrer une représentation en vue d'oiseau à son caractère fictionnel: Ramusio nous rappelle qu'un point de vue vertical sur le monde ne peut être que le résultat d'un dispositif 
intellectuel. À celui-ci s'oppose donc le mode de représentation plus fréquemment employé par Ramusio, fondé sur l'expérience des hommes sur le monde et qui consiste en une représentation horizontale en prise directe avec celui-ci. Les deux dispositifs sont-ils incompatibles? Si toute prise en compte globale de la Terre relève de la spéculation, cela suppose-t-il que Ramusio exclut la possibilité d'une représentation de son intégralité?

\section{Image d'ensemble et géographie}

16 Après ce détour par une allusion brève et abstraite à l'ensemble de la Terre, il nous faut aborder la question plus spécifique de la représentation de la Terre offerte par les Navigationi et viaggi. En effet, le caractère spéculatif du modèle du globe terrestre et de la rotation du Soleil autour de celui-ci auquel Ramusio fait référence dans l'introduction du troisième volume ne résout pas la question de l'image d'ensemble livrée par la compilation. On pourrait faire l'hypothèse que ce discorso, par la présentation de ce dispositif intellectuel, offre une clé de lecture de la compilation; cependant, cette grille de compréhension du monde ne peut être que partiellement satisfaisante pour le lecteur des Navigationi et viaggi puisqu'elle ne lui offre pas de solution pour situer spatialement les lieux décrits: elle lui fournit simplement une explication concernant la variété dont témoignent les différents récits.

17 La seconde référence à la Terre comme globe dans les Navigationi et viaggi se trouve dans le discorso traitant du commerce des épices (Ramusio, 1554 fol. 409v.-415v). En effet, dans la mise en scène du dialogue qui constitue le cadre de ce discorso, Ramusio semble suggérer qu'il est possible de considérer l'ensemble de la Terre par le recours à sa représentation graphique sous forme sphérique par le biais de cet objet qu'il nomme "balla », c'est-à-dire « boule, sphère ». L'interlocuteur anonyme dont Ramusio rapporte les propos dans la seconde moitié de ce discorso illustre en effet sa démonstration en ayant recours à une sphère représentant le globe terrestre et sur laquelle il trace de la main les parcours dont il parle. Précisons que les parcours dont il est question dans ce discorso - les routes des épices - sont tous hypothétiques: la démonstration de l'interlocuteur de Ramusio, un mathématicien et philosophe anonyme, vise en effet à prouver qu'il existe des routes alternatives à celles existantes, et donc qu'une concurrence au monopole portugais est possible.

18 Comme il est explicité dans l'introduction au troisième volume, tout regard surplombant ne peut être qu'une vue de « l'œil de l'intellect », or les explorations dont Ramusio compile les récits ont démontré que cette représentation spéculative était jusque-là incomplète. En effet, le rare cas où dans cette compilation est envisagée une représentation de l'intégralité du globe terrestre consiste en fait à identifier les zones où la connaissance géographique fait défaut: ces routes des épices alternatives devraient emprunter des zones encore non explorées de la surface terrestre. Ce discorso illustre donc l'usage possible des textes de la compilation, comme si le philosophe anonyme n'était autre que le lecteur idéal des Navigationi et viaggi. En effet, les propos de ce personnage prennent appui sur nombre des récits recueillis (il cite tour à tour Marco Polo, volume II, Jacques Cartier, Antonio de Mendoza, Marco da Nizza, Francisco Vásquez Coronado, volume III, et Juan Gaetano, volume I) : il les met en relation et en tire des conclusions en identifiant les espaces encore inconnus, c'est-à-dire les espaces qui n'ont pas été explorés et / ou dont aucune description n'est disponible. Ainsi, 
l'exposé du philosophe anonyme suppose que la lecture individuelle des textes doit être suivie d'un effort pour situer les espaces décrits les uns par rapport aux autres. Si la compilation ne propose aucune description complète de la Terre en une seule fois, elle en livre des descriptions partielles et, par l'exemple du philosophe anonyme, Ramusio semble suggérer que c'est à la charge du lecteur de procéder à leurs mises en relation.

Cette mise en relation des espaces entre eux correspond pleinement au savoir géographique tel qu'il est défini par Ptolémée, pour le distinguer de la chorographie. En effet, il établit dans la Géographie une comparaison entre ces savoirs et la peinture d'un portrait : la chorographie est cet art de peindre les parties indépendamment du tout, comme si l'on peignait un œil ou une oreille séparément, alors que la géographie a le souci de les inclure dans leur cadre global, c'est-à-dire comme un visage constitué de chacune de ses parties (Aujac, 1993). De la même façon, les récits de voyages décrivent les zones indépendamment les unes des autres, alors que la géographie les prend en compte en les incluant dans un "portrait » global de la Terre : même une description parcellaire a pour objectif la reconstitution d'une image d'ensemble. Ce modèle, appliqué aux Navigationi et viaggi, nous permet de distinguer entre la microstructure des récits, qui relève de la chorographie, et la macrostructure de la compilation, qui participe de la géographie. En rassemblant ces récits dans un seul ouvrage, et en les insérant dans une structure portante, Ramusio livre donc à la géographie le savoir chorographique de récits de voyages individuels. Comment s'effectue ce passage de la chorographie à la géographie? La mise en forme des récits de voyage dans la compilation des Navigationi et viaggi livre une représentation de l'espace selon la modalité du lien et non du fragment: les espaces décrits dans les récits de voyage ne sont pas des parcelles indépendantes mais les tasseaux d'un ensemble dont, on l'a dit, Ramusio considère que les contours complets sont encore inconnus de ses contemporains. Nous allons voir les modalités selon lesquelles des liens sont établis entre les différents espaces décrits.

\section{Espaces en relation}

Le dispositif par lequel sont mis en relation ces espaces est la structure d'ensemble de la compilation, où le matériau brut des récits de voyage est réparti en trois volumes. Or, cette répartition prend appui sur des critères qui ne sont pas uniquement des critères spatiaux, c'est-à-dire que les relations entre ces espaces ne sont pas définies uniquement par leur contiguïté physique. Ainsi, la tripartition du recueil ne reproduit pas une division géographique conventionnelle (parties ou régions) mais rend compte des processus d'exploration des lieux décrits. Le premier volume, où sont compilés des récits traitant à la fois de l'Afrique et de l'océan Indien, retrace les parcours d'exploration portugais dans ces zones, le deuxième est consacré à des espaces explorés par les Vénitiens (Proche-Orient, Asie et Europe du Nord) et le troisième à cette région du monde dont la connaissance a été rendue possible par les voyages espagnols, c'est-àdire les Caraïbes et le continent américain. Ainsi, lire les récits dans l'ordre dans lequel Ramusio les livre dans les Navigationi et viaggi revient à effectuer le même parcours que les explorations du siècle et demi qui précède la publication du recueil. Les espaces s'agencent donc autour des trajectoires prises par les voyageurs circulant dans des zones dont la connaissance a été rendue possible par les explorations pionnières de certaines puissances européennes. 
21 Même si les processus d'exploration et de contrôle de l'espace se déclinent de manière différente en fonction des puissances en question, l'organisation de la compilation permet toutefois de souligner (comme Ramusio le rappelle constamment dans les paratextes) que tout voyage exploratoire a lieu grâce aux initiatives permises - et directement financées, dans certains cas - par les gouvernants. La connaissance du monde dépend donc d'une volonté politique et ses processus suivent, de ce fait, un projet politique qui varie en fonction de chacune des puissances européennes. Mais l'organisation du recueil reflète également la conscience du caractère épistémologique de cette exploration du monde, au sens où ces projets politiques naissent comme ou deviennent des projets de connaissance du monde. Les figures du savant et du prince sont ainsi les deux figures centrales de la compilation, puisque celle-ci s'adresse d'abord à eux, comme l'indique Ramusio dans la dédicace du premier volume : « mais que dis-je du plaisir qu'en tireront les érudits, et les savants ; car qui pourrait douter du fait que bien des seigneurs, aussi, et des princes, ne se divertissent d'une telle lecture? ? (Ramusio, 1554 n.p.). Plutôt qu'un positionnement spatial des lieux, l'image livrée par la compilation dans son ensemble permet donc de rendre compte de la manière dont la connaissance de ces lieux a été rendue possible par - et pour - les hommes, en l'occurrence des Européens. L'espace géographique ainsi défini est à la fois politique et épistémologique.

Rendre compte de l'espace en fonction de l'action des hommes dans et sur celui-ci implique de plus qu'aucune représentation géographique ne puisse prétendre à une validité pérenne. Ce constat, qui lui aussi prend appui sur une comparaison entre le savoir géographique des Anciens et des Modernes (par exemple dans le cas du changement de toponymes, Ramusio, 1559 fol. 65r.), constitue une justification ultérieure au choix de la mise en forme par la compilation. En effet, cette structure permet une augmentation continue du matériau recueilli et rend possible, comme on l'a dit, une comparaison constante du contenu des descriptions en vue de la sélection des informations considérées comme pertinentes ou fiables.

Enfin, notons que Ramusio établit une distinction entre l'identification de la centralité de l'action humaine dans l'établissement du savoir géographique et le fait de ne rendre compte que de cette action; en effet, il refuse catégoriquement que le récit de voyage ne s'apparente qu'à une simple chronique. Ses attentes vis-à-vis du contenu d'un récit de voyage sont explicitées dans l'introduction au troisième volume, c'est-à-dire à propos du cas spécifique du continent américain où l'exploration et la conquête font l'objet de luttes entre factions qui prennent souvent le dessus, dans les récits, sur l'intérêt scientifique de leur contenu. En effet, nombre des relations espagnoles s'attachent à rapporter les faits d'armes des conquistadors plutôt qu'à procéder à des descriptions précises de la nature de ces régions :

Ce qui a constitué une grande perte pour les savants, qui désirent lire et comprendre dans le détail et qui préfèrent connaître les choses susdites que la nature produit dans ces régions, différentes de celles qui naissent chez nous, plutôt que les guerres civiles que les Espagnols ont menées entre eux [...] des choses naturelles susdites véritablement ils ne s'y intéressent que brièvement, dans la mesure où ils ne peuvent pas ne pas les nommer à l'occasion. (Ramusio, 1556 fol. 3v.)

24 Cette indication implique que la position traditionnellement ancillaire de la géographie par rapport à l'histoire - de la géographie comme description du cadre spatial des événements historiques - ne peut être appliquée au savoir géographique tel que le 
conçoit Ramusio (Descendre, 2010). De plus, ses para-textes indiquent comme les héros de son temps les explorateurs en tant qu'ils ont permis l'augmentation de la connaissance humaine : ce sont eux qui devraient obtenir les honneurs et pour lesquels on devrait ériger des statues (Ramusio, 1554 fol. 383r.).

\section{Conclusions}

Par le recours aux récits de voyage comme source première du savoir géographique, Ramusio confère à ce savoir une provenance fondamentalement expérimentale. Les risques qu'implique ce choix - le caractère aléatoire et individuel de l'expérience humaine et l'espace réduit que celle-ci peut couvrir - sont compensés par la mise en forme compilatoire qui permet une complémentarité entre les diverses expériences rapportées. Ainsi, le contenu et la structure des Navigationi et viaggi reposent sur un renversement de la hiérarchie ptoléméenne : le savoir géographique est fondé sur la description minutieuse des lieux dont les hommes peuvent faire l'expérience, la topographie et la chorographie constituent donc le point de départ de la géographie, et non l'inverse. De même, le détail précède l'image d'ensemble et l'expérience précède sa formalisation. Les deux seules apparitions de la Terre dans son intégralité visent à démontrer, d'une part, que la connaissance de celle-ci reste très lacunaire et, d'autre part, que la cosmographie peut servir avant tout à en expliquer la variété, et non la configuration spatiale. Le recours à un dispositif cosmographique dans l'introduction au troisième volume illustre toutefois la complémentarité entre géographie qualitative et géographie quantitative : si la première consiste en un recensement de la variété du monde, la seconde permet d'apporter une explication au constat de cette variété.

Cette proposition d'une géographie à grande échelle, où l'expérience des hommes est primordiale pour appréhender le monde, interroge la validité du modèle ptoléméen pour rendre compte du projet des Navigationi et viaggi. Le point commun entre Ramusio et l'auteur de la Géographie se trouve surtout dans le fait qu'ils mettent à disposition des éléments - des données chez Ramusio, une méthode et des coordonnées chez Ptolémée - permettant à d'autres d'établir un savoir géographique, que celui-ci soit formalisé dans des textes ou par des cartes. Les autres modèles antiques auxquels fait référence l'éditeur Giunti dans l'adresse aux lecteurs nous indiquent deux aspects supplémentaires de la géographie de Ramusio: d'une part, un intérêt pour les phénomènes naturels et leur compte rendu détaillé dans la lignée de Pline, à l'instar du texte de Gonzalo Fernández de Oviedo que Ramusio érige en exemple moderne; et, d'autre part, une attention pour la composante politique du savoir géographique, à l'image de Strabon. Cependant, la dimension politique du savoir géographique, qui apparait chez Strabon par la revendication d'une fonction formatrice de ce savoir pour les hommes politiques, se manifeste de manière différente chez Ramusio pour qui la géographie va de pair avec la politique parce que l'espace lui-même est politique, tout comme son appréhension. Ainsi, de par leur structure et de par leur inscription dans le contexte des conflits commerciaux et territoriaux entre les puissances européennes au milieu du $\mathrm{XVI}^{\text {e }}$ siècle, les Navigationi et viaggi identifient l'espace comme un objet de connaissance et de conquête qui ne peut être dissocié du politique. 


\section{BIBLIOGRAPHIE}

Aujac G., 1993, Claude Ptolémée, astronome, astrologue, géographe, Paris, éd. du CTHS, (427p.)

Besse J.-M., 2003, Les grandeurs de la Terre. Aspects du savoir géographique à la Renaissance, Lyon, ENS Éditions, (420 p.)

Bouloux N., 2002, Culture et savoirs géographiques en Italie au XIV ${ }^{e}$ siècle, Turnhout, Brepols, (340 p.)

Descendre R., 2010, « Dall'occhio della storia all'occhio della politica. Sulla nascita della geografia politica nel Cinquecento ", dans Mattioda E., Nascita della storiografia e organizzazione dei saperi. Atti del Convegno Internazionale di studi (Torino, 20-22 maggio 2009), Florence, Olschki, p. 155-179.

Hallyn F., 2008, Gemma Frisius, arpenteur de la terre et du ciel, Paris, Honoré Champion, (237 p.)

Headly J. M., 1997, « The Sixteenth-Century Venetian Celebration of the Earth Total Habitability: The Issue of the Fully Habitable World for Renaissance Europe », Journal of World History, 1997, $\mathrm{n}^{\circ} 8,1$, p. 1-27.

Lejosne F., 2017, « Viaggi di scoperte, racconti e pubblicazioni: le Navigationi et viaggi di Giovanni Battista Ramusio come opera di trasmissione ", dans Secchi Tarugi L., Viaggio e comunicazione nel Rinascimento : Atti del XXVII Convegno Internazionale (Chianciano Terme-Pienza, 16-18 luglio 2015), Florence, Franco Cesati editore, p. 337-349.

Milanesi M., 1984, Tolomeo sostituito. Studi di storia delle conoscenze geografiche nel XVI secolo, Milan, Unicopli, (251 p.)

Milanesi M., 1994, « Geography and Cosmography in Italy from XV to XVII Century », Memorie della Società Astronomica Italiana, Vol. 65, nº 2, p. 443-468.

Ramusio G. B., 1554-1556-1559, Navigationi et viaggi, Venise, Giunti, 3 vol.

Ramusio G. B., 1978-1988, Navigationi et viaggi, Milanesi M. (éd.), Turin, Einaudi, 6 vol.

Tessicini D., 2011, « Definitions of 'Cosmography' and 'Geography' in the Wake of Fifteenth- and Sixteenth-Century Translations and Editions of Ptolemy's Geography », dans Shalev Z. et Burnett C., Ptolemy's Geography in the Renaissance, Londres-Turin,The Warburg Institute-Nino Aragno Editore, p. 31-50.

\section{RÉSUMÉS}

Un parcours dans les para-textes des Navigationi et viaggi (Venise, 1550-1559) permet d'en identifier l'enjeu principal : se substituer à Ptolémée en collectant un savoir géographique fondé sur l'expérience des hommes dans le monde. Les témoignages de voyageurs constituent ainsi la principale matière de cet ouvrage publié par l'humaniste et secrétaire de la République de Venise Giovanni Battista Ramusio. Face aux limites potentielles de ses sources, en termes de quantité comme de qualité de l'information, Ramusio a recours à une forme permettant l'accumulation d'expériences considérées comme complémentaires : la compilation. Les textes sont regroupés et assemblés dans une même structure organique dans laquelle chaque récit individuel livre des informations de type chorographique. La représentation globale trouve sa place dans cette compilation sous deux formes différentes. Dans le discours de dédicace du troisième volume, elle est envisagée par le biais d'un dispositif cosmographique qui, par la spéculation, permet de saisir la variété du monde. Par ailleurs, à plusieurs reprises dans les para-textes, le recours à des 
représentations cartographiques sous la forme de globes permet d'illustrer les itinéraires empruntés par les explorations alors en cours, tout comme les opportunités futures de voyages. La macrostructure de la compilation fournit des indications sur la relation des espaces entre eux en reproduisant les trajectoires effectivement parcourues par les principales puissances européennes impliquées dans les explorations du premier âge moderne.

The main scope of Navigationi et viaggi (Venice, 1550-1559) can be identified through its paratexts: to provide a substitution for Ptolemy's work through the gathering of geographical knowledge based on human experience in the world. As such, travellers' testimonies represent the main material of this piece of work published by the humanist and secretary of the Republic of Venice Giovanni Battista Ramusio. But, because he is aware of the possible limits of his sources - both in qualitative and quantitative terms - Ramusio resorts to a form that allows the accumulation of experiences considered as complementary: a compilation. Texts are put together and assembled in this one and only organic structure in which each individual account delivers information of a chorographic type. In this compilation, globality is represented in two different ways. In the dedicatory discourse of the third volume, globality is considered through a cosmographical device which, thanks to a speculative approach, allows to fully grasp the variety of the world. Otherwise, more than once in the para-texts, the use of globe-shaped cartographical representations illustrates the itineraries of explorations which were then on-going, as well as the future travel opportunities. Furthermore, the macro-structure of the compilation delivers information on the organisation of spaces: it reproduces the trajectories followed by the European powers involved in the Age of discoveries.

\section{INDEX}

Mots-clés : Compilation, Venise, Ramusio, Giovanni Battista, Ptolémée, récits de voyage

Keywords : compilation, Venice, Ramusio, Giovanni Battista, Ptolemy, travel accounts

\section{AUTEUR}

\section{FIONA LEJOSNE}

Maîtresse de Conférences

Sorbonne Nouvelle

Département d'Études Italiennes et Roumaines

13, rue Santeuil

75231 PARIS CEDEX 05

fiona.lejosne@sorbonne-nouvelle.fr 